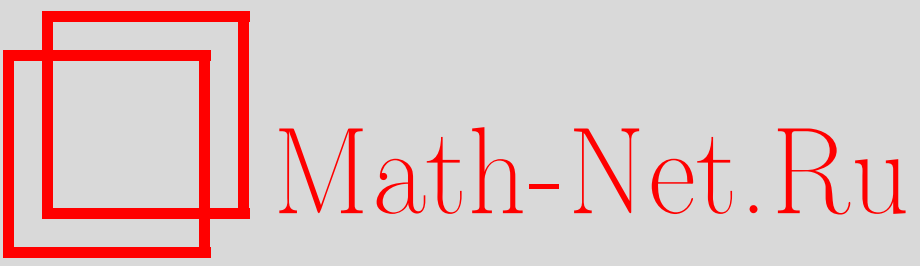

Н. А. Кудряшов, Нелинейные дифференциальные уравнения четвертого порядка с решениями в виде трансцендент, ТМФ, 2000, том 122, номер 1, 72-87

DOI: https://doi.org/10.4213/tmf556

Использование Общероссийского математического портала Math-Net.Ru подразумевает, что вы прочитали и согласны с пользовательским соглашением

http://www.mathnet.ru/rus/agreement

Параметры загрузки:

IP: 54.89 .56 .158

26 апреля 2023 г., 18:23:28 
ТЕОРЕТИЧЕСКАЯ

И МАТЕМАТИЧЕСКАЯ

ФИЗИКА

Том 122, № 1

январь, 2000

(C) 2000 г.

\author{
Н. А. Кудряшов*
}

\title{
НЕЛИНЕЙНЫЕ ДИФФЕРЕНЦИАЛЬНЫЕ УРАВНЕНИЯ ЧЕТВЕРТОГО ПОРЯДКА С РЕШЕНИЯМИ В ВИДЕ ТРАНСЦЕНДЕНТ
}

Представлены две иерархии обыкновенных дифференциальных уравнений и соотношение между ними. Найдены рациональные и специальные решения одной из иерархий. Показано, что решения двух дифференциальных уравнений являются существенно трансцендентными функциями от постоянных интегрирования.

\section{1. ВВЕДЕНИЕ}

Более ста лет назад Пенлеве и его ученики начали исследование дифференциальных уравнений второго порядка. Эти исследования были основаны на изучении сингулярностей решений уравнений. Пенлеве и его соавторы обнаружили, что из всех возможных уравнений определенного вида имеется только пятьдесят типов уравнений, у которых отсутствуют подвижные критические точки. Далее они показали, что сорок четыре из этих пятидесяти уравнений интегрируемы в терминах известных ранее функший (таких как эллиптические функции и решения линейных уравнений) или приводятся к одному из шести неприводимых нелинейных обыкновенных дифференциальных уравнений (ОДУ). Они также доказали, что имеется в точности шесть уравнений второго порядка, чьи решения дают новые функции. Эти функции называются теперь трансцендентами Пенлеве.

Позднее Бюро и Косгрове обобшили исследования Пенлеве и классифицировали другие уравнения второго и третьего порядков на основе возможных сингулярностей их решений, но они не нашли новых функций, подобных трансцендентам Пенлеве. Кстати, Пенлеве полагал, что среди решений уравнений третьего порядка не следует ожидать появления новых функций и что для их поиска следует перейти к исследованию уравнений четвертого порядка.

В недавних работах [1-3] нами была предпринята попытка решить задачу определения новых (помимо трансцендент Пенлеве) функций, определяемых решениями нелинейных ОДУ. Имеется несколько подходов к поиску уравнений, имеюших трансценденты,

\footnotetext{
* Московский инженерно-физический институт, Москва, Россия
} 
но мы воспользуемся простейшим, основанным на применении гипотезы Пенлеве, выдвинутой Абловицем, Рамани и Сигуром [4, 5], что каждое обыкновенное дифференциальное уравнение, возникающее как редукция полностью интегрируемого дифференциального уравнения в частных производных, является уравнением типа Пенлеве, возможно, после преобразования переменных. Обычно эта гипотеза используется как тест Пенлеве: если данное дифференциальное уравнение в частных производных приводимо к ОДУ, не являющемуся уравнением типа Пенлеве, то этот тест предсказьвает, что исходное дифференциальное уравнение в частных производных не является полностью интегрируемым $[4,5]$. С другой стороны, ОДУ, обладающее свойством Пенлеве, можно получить с помошью редукции из интегрируемого дифференциального уравнения в частных производных. Это обстоятельство позволяет искать ОДУ, имеюшие решения в виде трансцендентных функций от постоянных интегрирования.

Мы применили этот подход в работе [1] и получили две иерархии ОДУ вида

$$
d^{n+1}(u)=\frac{z}{2}, \quad n=1,2, \ldots,
$$

и

$$
\left(\frac{d}{d z}+2 v\right) d^{n}\left(v_{z}-v^{2}\right)-z v=\alpha .
$$

Оператор $d^{n}$ в уравнениях (1.1) и (1.2) определяется соотношениями Ленарда

$$
\begin{gathered}
\frac{d}{d z} d^{n+1}=d_{z z z}^{n}+2 u d_{z}^{n}+2 u_{z} d^{n} \\
d^{0}=\frac{1}{2}, \quad d^{1}=u .
\end{gathered}
$$

Следует отметить, что иерархии (1.1) и (1.2) обладают замечательными свойствами. Полагая $n=1$, из уравнений (1.1) и (1.2) получаем первое и второе уравнения Пенлеве:

$$
\begin{gathered}
u_{z z}+3 u^{2}=\frac{z}{2}, \\
v_{z z}=2 v^{3}+z v+\alpha .
\end{gathered}
$$

Взяв $n=2$ в уравнениях (1.1) и (1.2), получим уравнения четвертого порядка:

$$
u_{z z z z}+5 u_{z}^{2}+10 u u_{z z}+10 u^{3}=\frac{z}{2}
$$

$$
v_{z z z z}=10 v^{2} v_{z z}+10 v v_{z}^{2}-6 v^{5}+z v+\alpha
$$

Хорошо известно, что уравнения (1.4) определяют функции нового типа, являюшиеся трансцендентами Пенлеве. Возникает вопрос, являются ли решения уравнений (1.5), (1.6) трансцендентными функциями от постоянных интегрирования.

Уравнения (1.5) и (1.6) проверены нами на тест Пенлеве и обнаружено, что они проходят его [2]. Для уравнений (1.1) и (1.2) в [2] найдены лаксовы пары. Оказалось, что решения уравнений (1.5) и (1.6) являются сушественно трансцендентными функциями от 
констант интегрирования [3]. Кроме того, есть основания полагать, что трансценденты, определяемые уравнениями (1.5) и (1.6), являются новыми функциями, поскольку можно ожидать, что уравнения (1.5) и (1.6) неприводимы.

В данной работе мы ставим своей целью ввести две новые иерархии ОДУ и показать, что они имеют свойства, подобные уравнениям (1.1) и (1.2).

Работа построена следующим образом. Две иерархии, которые мы собираемся изучать, вводятся в разделе 2. Результаты исследования двух уравнений четвертого порядка на тест Пенлеве приведены в разделе 3 . Соотношения между этими иерархиями даны в разделе 4 , где мы получим некоторые рациональные и специальные решения уравнений четвертого порядка. Доказательство того, что решения этих уравнений являются существенно трансцендентными функциями относительно констант интегрирования, приведено в разделе 5 .

\section{2. ИССЛЕДУЕМЫЕ ИЕРАРХИИ}

Для того чтобы ввести две новые интегрируемые иерархии ОДУ, воспользуемся уравнениями Кодри-Додда-Гиббона и Каупа-Купершмидта, которые имеют вид [6-10]

$$
u_{t}+\Theta_{1} G_{n}(u)=0, \quad n=1,2, \ldots,
$$

и

$$
a_{t}+\Theta_{2} H_{n}(a)=0, \quad n=1,2, \ldots,
$$

где операторы $\Theta_{1}$ и $\Theta_{2}$ определены формулами

$$
\Theta_{1}=\Theta_{2}=D^{3}+2 u D+u_{x}, \quad D=\frac{\partial}{\partial x},
$$

а операторы $G_{n}$ и $H_{n}$ находятся из рекуррентных соотношений [9]

$$
G_{n+2}=J_{1}(u) \Theta_{1}(u) G_{n}
$$

и

$$
H_{n+2}=J_{2}(a) \Theta_{2}(a) H_{n}
$$

при условии, что

$$
\begin{array}{ll}
G_{0}=1, & G_{1}=u_{x x}+\frac{1}{4} u^{2}, \\
H_{0}=1, & H_{1}=a_{x x}+4 a^{2} .
\end{array}
$$

Операторы $J_{1}$ и $J_{2}$ определены формулами

$$
J_{1}=D^{3}+\frac{1}{2} D^{2} u D^{-1}+\frac{1}{2} D^{-1} u D^{2}+\frac{1}{8}\left(u^{2} D^{-1}+D^{-1} u^{2}\right)
$$


(где $\left.D^{-1}=\int d x\right)$ и

$$
J_{2}=D^{3}+3(u D+D u)+2\left(D^{2} u D^{-1}+D^{-1} u D^{2}\right)+8\left(u^{2} D^{-1}+D^{-1} u^{2}\right) .
$$

Модифишированные уравнения для (2.1) и (2.2) можно представить в виде

$$
\begin{aligned}
W_{t}+D(D+W) G_{n}\left(W_{x}-\frac{1}{2} W^{2}\right) & =0, \\
V_{t}+D(D+V) H_{n}\left(V_{x}-\frac{1}{2} V^{2}\right) & =0 .
\end{aligned}
$$

ЗАМЕчАниЕ 2.1. Уравнения (2.10) и (2.11) совпадают при замене переменных $W=$ $2 \mathrm{~V}$, так что эти уравнения, вообще говоря, не различаются.

Применение теста Пенлеве к уравнениям (2.1) и (2.2) ведет к уравнениям сингулярных многообразий, которые можно записать в виде $[9,10]$

$$
Y_{t}+Y_{x} H_{n}(\{Y ; x\})=0
$$

и

$$
\Phi_{t}+\Phi_{x} G_{n}(\{\Phi ; x\})=0,
$$

где $\{Y ; x\}-$ производная Шварца [9],

$$
\{Y ; x\}=\frac{Y_{x x x}}{Y_{x}}-\frac{3}{2} \frac{Y_{x x}^{2}}{Y_{x}^{2}} .
$$

Легко видеть, что уравнения (2.1), (2.2), (2.10)-(2.13) допускают группу преобразований неоднородного растяжения, поэтому их решения можно искать, используя автомодельные переменные. Если взять

$$
Y(x, t)=t^{\frac{m_{n}}{2 n+1}} \varphi(z), \quad z=x[(2 n+1) t]^{-\frac{1}{2 n+1}},
$$

то из уравнений (2.12) получаем иерархию ОДУ в виде

$$
\varphi_{z} h_{n}(\{\varphi ; z\})-z \varphi_{z}+m_{n} \varphi=0,
$$

где оператор $h_{n}$ определен формулами $(2.5),(2.7)$ и (2.9) в соответствии с операторами $H_{n}, \Theta_{2}$ и $J_{2}$ при $x \rightarrow z$.

Аналогично, принимая во внимание автомодельные переменные

$$
\Phi(x, t)=t^{\frac{P_{n}}{2 n+1}} \Psi(z), \quad z=x[(2 n+1) t]^{-\frac{1}{2 n+1}},
$$

из уравнений (2.13) можно получить иерархию ОДУ в виде

$$
\Psi_{z} g_{n}(\{\Psi ; z\})-z \Psi_{z}+p_{n} \Psi=0 .
$$

Оператор $g_{n}$ в (2.18) определен в соответствии с операторами $G_{n}, \Theta_{1}$ и $J_{1}$ при $x \rightarrow z$. 
Полагая $m_{n}=0$ в уравнениях (2.16), получаем иерархию ОДУ

$$
h_{n}(F)=z,
$$

если ввести обозначение

$$
F=\{\varphi ; z\}
$$

Из уравнения (2.19) при $n=1$ получаем первое уравнение Пенлеве

$$
F_{z z}+4 F^{2}=z
$$

Полагая $n=2$ в (2.19), можно получить уравнение четвертого порядка

$$
F_{z z z z}+12 F F_{z z}+2 F_{z}^{2}+\frac{32}{3} F^{3}=z .
$$

ЗАмечАниЕ 2.2. Выбор $p_{n}=0$ в уравнениях (2.18) после замены переменных также приводит к уравнению (2.19).

С другой стороны, можно видеть, что уравнение (2.22) отличается от рассмотренного ранее [1] уравнения (1.5). Под действием оператора $\left(\varphi_{z}\right)^{-1}(d / d z)$ последовательность ОДУ (2.16) принимает вид

$$
\left(\frac{d}{d z}+\omega\right) h_{n}\left(\omega_{z}-\frac{1}{2} \omega^{2}\right)-z \omega+\beta_{n}=0
$$

если учесть, что

$$
\omega=\frac{\varphi_{z z}}{\varphi_{z}}, \quad \beta_{n}=m_{n}-1 .
$$

Иерархия ОДУ (2.23) отлична также и от последовательностей ОДУ (1.2), обсуждаемых в [1]. Она может быть получена из уравнений (2.11) с использованием автомодельных переменных.

При $n=1$ в (2.23) мы имеем уравнение четвертого порядка

$$
\omega_{z z z z}+5 \omega_{z} \omega_{z z}-5 \omega^{2} \omega_{z z}-5 \omega \omega_{z}^{2}+\omega^{5}-z \omega+\beta_{n}=0
$$

Следует отметить, что уравнения (2.19) и (2.23) были получены редукцией интегрируемого дифференциального уравнения в частных производных к ОДУ, и поэтому можно ожидать, что эти последовательности ОДУ являются интегрируемыми, поскольку в соответствии с гипотезой Абловитца, Рамани и Сегура они должны обладать свойством Пенлеве. Любопытно отметить, что имеются две последовательности ОДУ (1.1) и (2.19), дающие первое уравнение Пенлеве при $n=1$, но в общем случае эти иерархии яВляются различными. 


\section{3. ТЕСТ ПЕНЛЕВЕ ДЛЯ УРАВНЕНИЙ (2.22) И (2.25)}

В этом разделе мы хотим применить тест Пенлеве к уравнениям (2.22) и (2.25) с целью определить поведение подвижных критических точек в обших решениях этих уравнений.

Для исследования таких уравнений мы используем тест Пенлеве для ОДУ, представленный в $[4,11]$. Суть метода состоит в следующем.

Пусть дано ОДУ

$$
E(u, z)=0 .
$$

При этом мы имеем в виду уравнения (2.22) и (2.25).

В первую очередь, мы ишем возможные семейства решений уравнения (3.1), полагая

$$
u \cong \frac{u_{0}}{\left(z-z_{0}\right)^{p}}
$$

где $p$ - порядок сингулярности, $z_{0}$ - подвижная сингулярность, а $u_{0}$ - константы, которые следует определить после подстановки (3.2) в уравнение (3.1).

Первое необходимое условие отсутствия подвижных критических сингулярностей требует целочисленности всех $p$ (см. $[11,12])$.

Возьмем уравнение (2.22) в виде

$$
F_{z z z z}+\frac{3}{2} F F_{z z}+\frac{3}{4} F_{z}^{2}+\frac{1}{6} F^{3}-z=0
$$

Подстановка (3.2) в уравнение (3.3) показывает, что это уравнение имеет два семейства решений с $\left(p, u_{0}\right):(-2,-12)$ и $(-2,-60)$.

Уравнение $(2.25)$ в виде

$$
\omega_{z z z z}+\frac{5}{2} \omega_{z} \omega_{z z}-\frac{5}{4} \omega^{2} \omega_{z z}-\frac{5}{4} \omega \omega_{z}^{2}+\frac{1}{16} \omega^{5}-z \omega+\beta_{n}=0
$$

допускает четыре семейства решений $\left(p, u_{0}\right):(-1,-4),(-1,2),(-1,8)$ и $(-1,-6)$. Очевидно, что первое необходимое условие интегрируемости уравнений (3.3) и (3.4) выполнено.

Второе необходимое условие отсутствия подвижных критических точек в общем решении уравнения (3.1) требует, чтобы степень $N$ в уравнении (3.1) равнялась числу индексов Фукса и все $N$ этих индексов всех семейств были различными целыми $[10,11]$.

Подставляя

$$
u \cong \frac{u_{0}}{\left(z-z_{0}\right)^{p}}+u_{j}\left(z-z_{0}\right)^{j-p}
$$

в уравнение (3.1) и приравнивая к нулю выражения при одной и той же степени $u_{j}$, получим полиномиальное уравнение для определения индексов Фукса.

Подстановка (3.5) в уравнение (3.3) с $u_{0}=-12$ и $p=-2$ дает уравнение

$$
j^{4}-14 j^{3}+53 j^{2}-28 j-96=0,
$$


которое имеет четыре различных корня - индексы Фукса $j_{1}=-1, j_{2}=3, j_{3}=4$ и $j_{4}=$ 8 .

Другое семейство решений уравнения (3.3) с $u_{0}=-60$ и $p=-2$ приводит к уравнению

$$
j^{4}-14 j-19 j^{2}+476 j+480=0
$$

которое дает следуюшие индексы Фукса: $j_{1}=-1, j_{2}=-5, j_{3}=8$ и $j_{4}=12$.

Можно видеть, что второе необходимое условие теста Пенлеве для уравнения (3.3) также вьполнено.

Рассмотрим теперь второе необходимое условие для уравнения (3.4). Подставляя (3.5) в уравнение (3.4) и приравнивая к нулю выражения при одной и той же степени $u_{j}$, получаем одно и то же уравнение для индексов Фукса при $u_{0}=-4$ и $u_{0}=2$ :

$$
j^{4}-10 j^{3}+25 j^{2}-36=0
$$

Уравнение (3.8) имеет корни $j_{1}=-1, j_{2}=2, j_{3}=3$ и $j_{4}=6$.

В случае $u_{0}=-6$ получаем уравнение

$$
j^{4}-10 j^{3}+5 j^{2}+100 j+84=0
$$

которое дает индексы Фукса $j_{1}=-1, j_{2}=-2, j_{3}=6$ и $j_{4}=7$. При $u_{0}=8$ имеем полиномиальное уравнение в виде

$$
j^{4}-10 j^{3}-65 j^{2}+450 j+504=0
$$

корни которого равны $j_{1}=-1, j_{2}=-7, j_{3}=6$ и $j_{4}=12$.

Этот результат показывает, что второе необходимое условие отсутствия подвижных критических точек в общем решении уравнения (3.4) также выполнено.

Третье необходимое условие - сушествование ряда Лорана для решения уравнения (3.1). Проверка этого условия для семейства с положительными индексами сводится к подстановке выражения

$$
u=\sum_{i=0}^{\infty} u_{i}\left(z-z_{0}\right)^{i-p}
$$

в уравнение (3.1) и к последующей проверке того, что $u_{j_{k}}\left(j_{k}-\right.$ индексы Фукса) могут быть выбраны в виде произвольных постоянных.

Для семейства (3.6) с положительными индексами Фукса из уравнения (3.3) получается разложение вида

$$
\begin{aligned}
F= & -12\left(z-z_{0}\right)^{-2}+a_{3}\left(z-z_{0}\right)+a_{4}\left(z-z_{0}\right)^{2}- \\
& -\frac{1}{4}\left(\frac{a_{3}^{2}}{4}+\frac{z_{0}}{21}\right)\left(z-z_{0}\right)^{4}-\frac{1}{16}\left(a_{3} a_{4}+1\right)\left(z-z_{0}\right)^{5}+ \\
& +a_{8}\left(z-z_{0}\right)^{6}+\frac{a_{3}}{300}\left(\frac{z_{0}}{7}+\frac{7}{12} a_{3}^{2}\right)\left(z-z_{0}\right)^{7}+\cdots
\end{aligned}
$$


В этом разложении $z_{0}, a_{3}, a_{4}$ и $a_{8}$ являются произвольными константами, и третье необходимое условие для этого семейства решений выполнено.

Для семейства (3.8) с положительными индексами Фукса из уравнения (3.4) следуют два разложения:

$$
\begin{aligned}
\omega= & -4\left(z-z_{0}\right)^{-1}+a_{2}\left(z-z_{0}\right)+a_{3}\left(z-z_{0}\right)^{2}+\left(\frac{z_{0}}{5}-\frac{7 a_{2}^{2}}{4}\right)\left(z-z_{0}\right)^{3}- \\
& -\frac{1}{4}\left(5 a_{2} a_{3}-\frac{\beta_{n}+4}{9}\right)\left(z-z_{0}\right)^{4}+a_{6}\left(z-z_{0}\right)^{5}+ \\
& +\frac{1}{16}\left(21 a_{2}^{2} a_{3}-\frac{71 a_{2}}{90}-\frac{7 a_{3} z_{0}}{5}-\frac{2 \beta_{n} a_{2}}{9}\right)\left(z-z_{0}\right)^{6}+\cdots
\end{aligned}
$$

и

$$
\begin{aligned}
\omega= & 2\left(z-z_{0}\right)^{-1}+a_{2}\left(z-z_{0}\right)+a_{3}\left(z-z_{0}\right)^{2}+\frac{1}{2}\left(\frac{a_{2}^{2}}{4}-\frac{z_{0}}{5}\right)\left(z-z_{0}\right)^{3}+ \\
& +\frac{\left(\beta_{n}-2\right)}{36}\left(z-z_{0}\right)^{4}+a_{6}\left(z-z_{0}\right)^{5}+ \\
& +\frac{1}{16}\left(\frac{a_{2}^{2} a_{3}}{8}-\frac{a_{3} z_{0}}{10}-\frac{5 \beta_{n} a_{2}}{72}-\frac{7 a_{2}}{180}\right)\left(z-z_{0}\right)^{6}+\cdots .
\end{aligned}
$$

Можно видеть, что $z_{0}, a_{2}, a_{3}$ и $a_{6}$ являются произвольными константами, и поэтому третье необходимое условие для решений уравнения (3.4) также выполнено. Тем самым уравнения (2.22) и (2.25) проходят тест Пенлеве для семейства решений с положительными индексами. Исследование необходимых условий для семейств решений с отрицательными индексами Фукса из-за значительного объема вычислений здесь не приводится, но они также были нами проверены с использованием метода возмушений Фукса, предложенного в [12].

\section{4. РАЦИОНАЛЬНЫЕ И СПЕЦИАЛЬНЫЕ РЕШЕНИЯ УРАВНЕНИЯ (2.25)}

Известно, что модифицированное уравнение для уравнений Кодри-Додда-Гиббона и Каупа-Купершмидта имеет два различных уравнения сингулярного многообразия [13, 14]. Используя автомодельные решения (2.15), получаем два соотношения для уравнения (2.25) и уравнений (2.16) и (2.18). Их можно записать в виде

$$
\begin{array}{r}
\left(\frac{d}{d z}+\omega\right) h_{n}\left(\omega_{z}-\frac{1}{2} \omega^{2}\right)-z \omega+m_{n}-1= \\
=\frac{1}{\varphi_{z}} \frac{d}{d z}\left[\varphi_{z} h_{n}(\{\varphi ; z\})-z \varphi_{z}+m_{n} \varphi\right]
\end{array}
$$

где

$$
\omega=\frac{\varphi_{z z}}{\varphi_{z}}
$$


и

$$
\begin{aligned}
& \left(\frac{d}{d z}+\omega\right) h_{n}\left(\omega_{z}-\frac{1}{2} \omega^{2}\right)-z \omega-\frac{p_{n}}{2}+\frac{1}{2}= \\
& =-\frac{1}{2 \Psi_{z}} \frac{d}{d z}\left[\Psi_{z} g_{n}(\{\Psi ; z\})-z \Psi_{z}+p_{n} \Psi\right]
\end{aligned}
$$

где

$$
\omega=-\frac{\Psi_{z z}}{2 \Psi_{z}}
$$

Используя предложенный в [14] подход к получению рациональных и специальных решений нелинейного дифференциального уравнения в частных производных, можно получить итерационные формулы Вайса [9] в виде

$$
\Phi_{n+1, x}=\frac{Y_{n}^{4}}{Y_{n, x}^{2}}
$$

И

$$
Z_{k+1, x}=\frac{\Phi_{k}}{\Phi_{k, x}^{\frac{1}{2}}}
$$

где $Y(x, t)$ и $\Phi(x, t)$ являются решениями уравнений $(2.12)$ и $(2.13)$.

Полагая [15], что

$$
\begin{aligned}
Y_{n}(x, t) & =t^{\frac{m_{n}}{2 n+1}} \varphi_{n}(z), \quad z=x[(2 n+1) t]^{-\frac{1}{2 n+1}}, \\
Y_{k+1}(x, t) & =t^{\frac{M_{n}}{2 n+1}} \varphi_{n}(z), \\
\Phi_{k}(x, t) & =t^{\frac{p_{k}}{2 k+1}} \Psi_{k}(z), \quad z=x[(2 n+1) t]^{-\frac{1}{2 n+1}}, \\
\Phi_{n+1}(x, t) & =t^{\frac{P_{n}}{2 n+1}} \Psi_{n+1}(z),
\end{aligned}
$$

и подставляя (4.7)-(4.10) в преобразования (4.5) и (4.6), получаем

$$
\Psi_{n+1, z}=\frac{\varphi_{n}^{4}}{\varphi_{n, z}^{2}}
$$

и

$$
\varphi_{k+1, z}=\frac{\Psi_{k}}{\Psi_{k, z}^{\frac{1}{2}}}
$$

при

$$
M_{k}=\frac{p_{k}+3}{2}, \quad P_{n}=2 m_{n}+3 .
$$

Постоянные $M_{k}$ и $P_{n}$, определенные формулами (4.13), отвечают новым значениям параметров в уравнениях (2.16) и (2.18).

Соотношения (4.1), (4.2) и формулы (4.11) и (4.12) можно использовать для получения рациональных и специальных решений уравнений (2.16) и (2.18), а потому и уравнений (2.23). 
Без потери общности найдем рациональные и специальные решения уравнения (2.25), пользуясь уравнениями (2.16) и (2.18) при $n=1$. В этом случае они принимают вид

$$
\varphi_{z}\left[\{\varphi ; z\}_{z z}+4\{\varphi ; z\}^{2}\right]-z \varphi_{z}+m_{n} \varphi=0
$$

и

$$
\Psi_{z}\left[\{\Psi ; z\}_{z z}+\frac{1}{4}\{\Psi ; z\}^{2}\right]-z \Psi_{z}+p_{n} \Psi=0 .
$$

Решения уравнения (2.25) можно получить в виде

$$
\omega=\frac{\varphi_{z z}}{\varphi_{z}}, \quad \omega=-\frac{\Psi_{z z}}{2 \Psi_{z}}
$$

Очевидно, что при $m_{n}=p_{n}=1$ имеем простейшие решения $\varphi_{0}=\Psi_{0}=z$ уравнений (4.14) и (4.15), что соответствует тривиальному решению $\omega_{0}=0$ уравнения (2.25) при $\beta_{0}=0$.

С использованием $\varphi_{0}$ и $\Psi_{0}$ мы можем найти рациональные решения уравнений (4.14) и (4.15) из итерационных формул

$$
\Psi_{n+1}=\int \frac{\varphi_{n}^{4}}{\varphi_{n, z}^{2}} d z, \quad \varphi_{k+1}=\int \frac{\Psi_{k}}{\Psi_{k, z}^{\frac{1}{2}}} d z
$$

Имеем

$$
\varphi_{1}=\frac{1}{2} z^{2}, \quad m_{n}=2, \quad \omega(z, 1)=z^{-1}
$$

и

$$
\Psi_{1}=\frac{1}{5}\left(z^{5}+36\right), \quad p_{n}=5, \quad \omega(z,-2)=-2 z^{-1} .
$$

Далее получаем

$$
\varphi_{2}=\frac{z^{5}-144}{20 z}, \quad m_{n}=4, \quad \omega(z, 3)=\frac{3 z^{6}-72}{z^{5}+36}
$$

$$
\Psi_{2}=\frac{1}{112} z^{7}, \quad p_{n}=7, \quad \omega(z,-3)=-3 z^{-1} .
$$

Аналогичным образом можно найти

$$
\begin{gathered}
\varphi_{3}=z^{5}-504, \quad m_{n}=5, \quad \omega(z, 4)=4 z^{-1} \\
\Psi_{3}=\frac{z^{11}}{70400}-\frac{27 z^{6}}{1600}+\frac{10611}{400} z+\frac{3645 z}{4\left(z^{5}+36\right)}, \quad p_{n}=11
\end{gathered}
$$

и

$$
\Psi_{4}=\frac{1}{325} z^{13}-\frac{252}{25} z^{8}+\frac{508032}{25} z^{3}+\frac{256048128}{25} z^{-2}-\frac{9217732608}{35} z^{-7}, \quad p_{n}=13 .(4.24)
$$

После подстановки $(4.23)$ и $(4.24)$ в $(4.16)$ получаем $\omega(z,-5)$ и $\omega(z,-6)$. 
Из (4.13) видно, что рациональные решения уравнения (2.25) можно получить для всех положительных и отрицательных целых значений параметра $\beta_{n}$ :

$$
\beta_{n}=n, \quad n \neq 2 \pm 3 k \quad(k=0,1,2, \ldots) .
$$

Рассмотрим теперь специальные решения уравнения (2.25). Заметим, что первое уравнение Пенлеве (2.21) получается из уравнений (4.14) и (4.15) при $m_{n}=p_{n}=0$, если положить

$$
F=\omega_{z}-\frac{1}{2} \omega^{2}=\{\varphi ; z\} .
$$

Поэтому при известном решении уравнения (2.21) можно найти специальное решение $\omega\left(z, \beta_{n}\right)$ из уравнения Риккатти (4.26). Из (4.16) находятся $\varphi(z)$ и $\Psi(z)$. Используя формулы (4.17), можно найти специальные решения уравнения (2.25) при других значениях параметра $\beta_{n}$. Эти значения параметра $\beta_{n}$ определяются формулами

$$
\beta_{n}=\frac{1}{2} \pm 3 k \quad(k=0,1,2, \ldots)
$$

и

$$
\beta_{n}=2 \pm 3 k \quad(k=0,1,2, \ldots) .
$$

Таким образом, рациональные и специальные решения уравнения (2.25) находятся для всех положительных и отрицательных значений параметра $\beta_{n}$ и для полуцелых значений, определенных формулой (4.27).

\section{5. ТРАНСЦЕНДЕНТЫ, ОПРЕДЕЛЯЕМЫЕ УРАВНЕНИЕМ (2.22)}

Учитывая отмеченные выше свойства уравнений (2.22) и (2.25), можно ожидать, что эти уравнения имеют свойство Пенлеве.

Решение задачи нахождения новых функций, определяемых нелинейными ОДУ (2.22) и (2.25), сводится теперь к исследованию функциональной зависимости их обшего решения от констант интегрирования. При этом возможны три различных случая [16-18].

В первом случае обшее решение уравнения имеет рациональную или алгебраическую зависимость от произвольных постоянных. Этот случай не приводит к новым функциям.

Во втором случае общее решение уравнения не имеет рациональной или алгебраической зависимости от произвольных постоянных, но произвольные постоянные могут входить в алгебраическую форму первого интеграла. В этом случае обшее решение есть полутрансцендентная функция постоянных интегрирования и новые функции также не возникают.

Третий случай соответствует специальной зависимости обшего решения уравнения от постоянных интегрирования. Он противоречит зависимости обшего решения уравнения от постоянных интегрирования в первом и втором случаях. Именно в этом случае получаются существенно трансцендентные функции от постоянных интегрирования.

Докажем, что обшие решения уравнений (2.22) и (2.25) являются сушественно трансцендентными функциями от постоянных интегрирования. Без потери общности рассмотрим уравнение (2.22), поскольку доказательство для уравнения (2.25) можно провести аналогичным образом. Можно сформулировать следующую теорему. 
ТеОрема 5.1. Общее решение уравнения (2.22) является существенно трансцендентной функиией своих постоянных интегрирования.

ДокАЗАТЕЛьство. Доказательство разбивается на две части. В первую очередь нам надо доказать, что общее решение имеет трансцендентную зависимость от начальных условий. Затем следует доказать, что общее решение уравнения (2.22) не является полутрансцендентной функцией. С помощью переменных $[16,17]$

$$
F=\lambda^{-2} F^{\prime}, \quad z=\lambda z^{\prime},
$$

где $\lambda$ - некоторый параметр, уравнение (2.22) можно преобразовать к виду

$$
F_{z z z z}+12 F F_{z z}+6 F_{z}^{2}+\frac{32}{3} F^{3}-\lambda^{7} z=0
$$

(штрихи у переменных опущены).

При $\lambda=0$ уравнение (5.2) принимает вид

$$
F_{z z z z}+12 F F_{z z}+6 F_{z}^{2}+\frac{32}{3} F^{3}=0 .
$$

Решение уравнения (5.3) имеет трансцендентную зависимость от произвольных постоянных. Однако оно имеет первьй интеграл вида

$$
P_{1}=F_{z} F_{z z z}-\frac{1}{2} F_{z z}^{2}+6 F F_{z}^{2}+\frac{8}{3} F^{4}=C_{1}
$$

Поэтому общее решение уравнения (5.3) является полутрансцендентной функцией от постоянных интегрирования.

Покажем, что у (2.22) (или уравнения (5.2) при $\lambda \neq 0$ ) нет первого интеграла полиномиального вида.

Предположим, что уравнение (2.22) имеет первый интеграл

$$
P=P\left(y, y_{1}, y_{2}, y_{3}, z\right)=C,
$$

где

$$
y=F, \quad y_{1}=F_{z}, \quad y_{2}=F_{z z}, \quad y_{3}=F_{z z z} .
$$

Тогда принимая во внимание определение интеграла (5.5), получаем уравнение вида

$$
E=\frac{\partial P}{\partial z}+\frac{\partial P}{\partial y} y_{1}+\frac{\partial P}{\partial y_{1}} y_{2}+\frac{\partial P}{\partial y_{2}} y_{3}+\frac{\partial P}{\partial y_{3}} y_{4}=0 \text {. }
$$

Уравнение (5.6) должно соответствовать уравнению (2.22), так чтобы было

$$
E=\left(y_{4}+12 y y_{2}+6 y_{1}^{2}+\frac{32}{3} y^{3}-z\right) Q,
$$

где $Q$ - многочлен от $y, y_{1}, y_{2}, y_{3}$ и $z$. 
Из (5.6) и (5.7) вытекает равенство

$$
\frac{\partial P}{\partial y_{3}}=Q
$$

Поэтому уравнение (5.7) принимает вид

$$
\frac{\partial P}{\partial z}+\frac{\partial P}{\partial y} y_{1}+\frac{\partial P}{\partial y_{1}} y_{2}+\frac{\partial P}{\partial y_{2}} y_{3}=\frac{\partial P}{\partial y_{3}}\left(12 y y_{2}+6 y_{1}^{2}+\frac{32}{3} y^{3}-z\right)
$$

Предположим теперь, что первый интеграл уравнения (2.22) имеет полиномиальный вид

$$
P=\sum_{k=0}^{m} r_{k} y_{3}^{m-k}
$$

где

$$
r_{k}=r_{k}\left(y, y_{1}, y_{2}, z\right) .
$$

Подставляя (5.10) в уравнение (5.9) и приравнивая к нулю одинаковые степени $y_{3}$, получаем следуюшую цепочку уравнений:

$$
\begin{gathered}
\frac{\partial r_{0}}{\partial y_{2}}=0 \\
\frac{\partial r_{1}}{\partial y_{2}}+\frac{\partial r_{0}}{\partial x}+\frac{\partial r_{0}}{\partial y} y_{1}+\frac{\partial r_{0}}{\partial y_{1}} y_{2}=0 \\
\frac{\partial r_{2}}{\partial y_{2}}+\frac{\partial r_{1}}{\partial z}+\frac{\partial r_{1}}{\partial y} y_{1}+\frac{\partial r_{1}}{\partial y_{1}} y_{2}=r_{0}\left(12 y y_{2}+6 y_{1}^{2}+\frac{32}{3} y^{3}-z\right), \\
\frac{\partial r_{k+1}}{\partial y_{2}}+\frac{\partial r_{k}}{\partial z}+\frac{\partial r_{k}}{\partial y} y_{1}+\frac{\partial r_{k}}{\partial y_{1}} y_{2}= \\
=(m-k+1) r_{k-1}\left(12 y y_{2}+6 y_{1}^{2}+\frac{32}{3} y^{3}-z\right) \quad(k=2, \ldots, m-1), \\
\frac{\partial r_{m}}{\partial z}+\frac{\partial r_{m}}{\partial y} y_{1}+\frac{\partial r_{m}}{\partial y_{1}} y_{2}=r_{m-1}\left(12 y y_{2}+6 y_{1}^{2}+\frac{32}{3} y^{3}-z\right) .
\end{gathered}
$$

Все коэффициенты $r_{k}, k=0,1, \ldots, m-1$, определяются из уравнений (5.12)-(5.15). При этом решения $r_{m-1}$ и $r_{m}$ должны удовлетворять уравнению (5.16), если $P$ является интегралом уравнения (2.22).

Из уравнения (5.12) имеем

$$
r_{0}=r_{0}\left(y, y_{1}, z\right) .
$$

Решение уравнения (5.13) можно представить в виде

$$
r_{1}=-\frac{1}{2} \frac{\partial r_{0}}{\partial y_{1}} y_{2}^{2}-b_{0} y_{2}+f_{1}\left(y, y_{1}, z\right)
$$

где

$$
b_{0}=\frac{\partial r_{0}}{\partial z}+\frac{\partial r_{0}}{\partial y} y_{1}
$$


Приравнивая одинаковые степени $y_{2}$ в уравнении (5.16), получаем

$$
\frac{\partial^{m+1} r_{0}}{\partial y_{1}^{m+1}}=0, \quad \frac{\partial^{m} b_{0}}{\partial y_{1}^{m}}=0 \text {. }
$$

Решая (5.12)-(5.16) и учитывая (5.20), можно найти обший вид полиномиальной зависимости $r_{0}$ от $y, y_{1}$ и $z$, однако заметим, что $r_{0}$ содержится в $r_{k}$ как линейное выражение, поэтому без потери обшности положим

$$
r_{0}=y_{1}^{m}
$$

Тогда можно записать

$$
r_{1}=-\frac{1}{2} m y_{1}^{m-1} y_{2}^{2}+f_{1}\left(y, y_{1}, z\right)
$$

и решение уравнения (5.14) принимает вид

$$
\begin{aligned}
r_{2}= & \frac{1}{8} m(m-1) y_{1}^{m-2} y_{2}^{4}+\frac{1}{2}\left(12 y y_{1}^{m}-\frac{\partial f_{1}}{\partial y_{1}}\right) y_{2}^{2}- \\
& -\left[\left(6 y_{1}^{2}+\frac{32}{3} y^{3}-z\right) y_{1}^{m}-\left(\frac{\partial f_{1}}{\partial z}+\frac{\partial f_{1}}{\partial y} y_{1}\right)\right] y_{2}+f_{2}\left(y, y_{1}, z\right) .
\end{aligned}
$$

Можно считать, что

$$
r_{k}=a_{k} y_{2}^{2 k}+b_{k} y_{2}^{2 k-2}+c_{k} y_{2}^{2 k-3}+\cdots
$$

где коэффициенты $a_{k}, b_{k}$ и $c_{k}$ зависят от $y, y_{1}$ и $z$.

Подставляя (5.24) в уравнение (5.15) и приравнивая выражения при одинаковых степенях $y_{2}$, получаем рекуррентные формулы

$$
\begin{aligned}
a_{k+1}= & -\frac{1}{2 k+2} \frac{\partial a_{k}}{\partial y_{1}}, \\
b_{k+1}= & \frac{1}{2 k}\left[(m-k+1) a_{k-i} 12 y-\frac{\partial b_{k}}{\partial y_{1}}\right], \\
c_{k+1}= & \frac{1}{2 k-1}\left[(m-k+1) a_{k-i}\left(6 y_{1}^{2}+\frac{32}{3} y^{3}-z\right)-\right. \\
& \left.-\frac{\partial c_{k}}{\partial y_{1}}-\left(\frac{\partial b_{k}}{\partial z}+y_{1} \frac{\partial b_{k}}{\partial y}\right)\right] .
\end{aligned}
$$

Из уравнения (5.16) имеем

$$
\begin{aligned}
\frac{\partial a_{m}}{\partial y_{1}} & =0 \\
\frac{\partial b_{m}}{\partial y_{1}} & =12 y a_{m-1} \\
\frac{\partial c_{m}}{\partial y_{1}}+\frac{\partial b_{m}}{\partial z}+y_{1} \frac{\partial b_{m}}{\partial y} & =\left(6 y_{1}^{2}+\frac{32}{3} y^{3}-z\right) a_{m-1} .
\end{aligned}
$$


Решения уравнений (5.25) и (5.29) принимают вид

так что

$$
a_{k}=(-1)^{k} \frac{m(m-1) \ldots(m-k+1)}{2^{k} k !},
$$

$$
a_{m-1}=(-1)^{m-1} \frac{m}{2^{m-1}} y_{1}, \quad a_{m}=(-1)^{m} \frac{1}{2^{m}} .
$$

Из (5.29) получаем, что

$$
b_{m}=(-1)^{m-1} \frac{m}{2^{m-1}}\left[6 y y_{1}^{2}+g_{1}(y, z)\right] .
$$

Подставляя (5.33) в уравнение (5.30) и приравнивая к нулю выражения при одинаковых степенях $y_{1}$, имеем

$$
g_{1}(y, z)=\frac{8}{3} y^{4}-z y+p_{1}(z)
$$

где $p_{1}(z)$ - некоторая функция $z$.

Методом математической индукции из уравнения (5.26) можно получить коэффициенты $b_{k}$ в виде

$$
b_{k}=(-1)^{k-1} \frac{m(m-1) \ldots(m-k+1)}{2^{k}(k-1) !} y_{1}^{m-k}\left[6 y y_{1}^{2}+\frac{8}{3} y^{4}-z y+p_{1}(z)\right] \text {. }
$$

Из уравнения (5.35) находим

$$
b_{1}=m y_{1}^{m-1}\left[6 y y_{1}^{2}+\frac{8}{3} y^{4}-z y+p_{1}(z)\right] .
$$

Учитывая решение (5.36), из уравнения (5.27) получаем

$$
c_{2}=-m y_{1}^{m-1}\left(\frac{\partial p_{1}}{\partial z}-u\right) \text {. }
$$

Полагая

$$
c_{k}=(-1)^{k-1} A_{k} y_{1}^{m-k+1}\left(\frac{\partial p_{1}}{\partial z}-y\right)
$$

(где $A_{k}$ являются некоторыми положительными константами), методом математической индукции получаем $c_{k+1}$ в виде

$$
c_{k+1}=(-1)^{k} A_{k+1} y_{i}^{m-k}\left(\frac{\partial p_{1}}{\partial z}-\frac{1}{2} y\right)
$$

где

$$
A_{k+1}=\frac{(m-k+1)}{(2 k-1)}\left[A_{k}+\frac{m(m-1) \ldots(m-k)}{2^{k-1}(k-1) !}\right] \text {. }
$$

Таким образом,

$$
c_{m}=(-1)^{m-1} A_{m} y_{1}\left(\frac{\partial p_{1}}{\partial z}-y\right)
$$

Подстановка (5.32), (5.33) и (5.41) в уравнение (5.30) приводит к противоречию:

$$
\left(\frac{m}{2^{m-1}}+A_{m}\right)\left(\frac{\partial p_{1}}{\partial z}-y\right) \neq 0
$$

Это противоречие показывает, что полиномиального первого интеграла для уравнения (2.22) нет и его решение является существенно трансцендентной функцией от своих постоянных интегрирования. Это доказывает теорему 5.1 . 


\section{6. ЗАКЛЮЧЕНИЕ}

Мы представили две новые иерархии нелинейных ОДУ и рассмотрели некоторые их свойства. Эти иерархии были получены с использованием автомодельных решений нелинейных точно решаемых дифференциальных уравнений в частных производных, и поэтому согласно гипотезе Абловица, Рамани и Сигура эти ОДУ также должны быть интегрируемыми. Мы применили тест Пенлеве для исследования уравнений четвертого порядка и проверили, что изучаемые уравнения проходят этот тест. Используя итерационные формулы (4.17) для одного из уравнений четвертого порядка, мы получили рациональные и специальные решения, сушествуюшие для всех целых и некоторых полуцелых значений параметра уравнения (2.25). Показано, что общее решение уравнения $(2.22)$ является существенно трансцендентной функцией от констант интегрирования.

Благодарности. Данная работа поддержана Международным научно-техническим центром, проект В23-96. Материал статьи частично основан на работе, поддержанной US Civilian Research and Development Foundation, грант № RG2-144.

\section{Список литературы}

[1] N. A. Kudryashov. Phys. Lett. A. 1997. V. 224. P. 353.

[2] N. A. Kudryashov, M. B. Soukharev. Phys. Lett. A. 1998. V. 237. P. 206.

[3] N. A. Kudryashov. J. Phys. A. 1998. V. 31. P. L129.

[4] M. J. Ablowitz, A. Ramani, H. Segur. J. Math. Phys. 1980. V. 21. P. 715, 1006.

[5] M. J. Ablowitz, P. A. Clarkson. Solitons, nonlinear evolution equations and inverse scattering. Cambridge: Cambridge Univ. Press., 1991.

[6] P. J. Caudrey, R. K. Dodd, J. D. Gibbon. Proc. Roy Soc. London. A. 1976. V. 351. P. 407.

[7] R. K. Dodd, J. D. Gibbon. Proc. Roy Soc. London. A. 1977. V. 358. P. 287.

[8] B. Kuperschmidt, G. Wilson. Invent. Math. 1981. V. 62. P. 403.

[9] J. Weiss. J. Math. Phys. 1984. V. 25. P. 13.

[10] J. Weiss. J. Math. Phys. 1986. V. 27. P. 1293.

[11] R. Conte. The Painlevé approach to nonlinear ordinary differential equations. In: The Painleve Property, One Century Later. CRM Series in Mathematical Physics. Ed. R. Conte. New York: Springer-Verlag, 1999. P. 77; solv-int/9710020.

[12] R. Conte, A. P. Fordy, A. Pickering. Physica D. 1993. V. 69. P. 33

[13] N. A. Kudryashov. J. Phys. A. 1994. V. 27. P. 2457.

[14] N. A. Kudryashov. J. Phys. A. 1997. V. 30. P. 5445.

[15] N. A. Kudryashov, A. Pickering. J. Phys. A. 1998. V. 31. № 47. P. 9505.

[16] Э. Л. Айнс. Обыкновенные дифференциальные уравнения. Харьков: ГНТИ Украины, 1939.

[17] В. И. Громак, Н. А. Лукашевич. Аналитические свойства решений уравнений Пенлеве. Минск: Университетское изд-во, 1990.

[18] В. В. Голубев. Лекции по аналитической теории дифференциальных уравнений. М.: ГИТТЛ, 1941. 